have an important influence in causing tallowy discoloration.

F. BISHOP

${ }^{2}$ Stricks, W., and Kolthoff, I. M., J. Amer. Chem. Soc, , 73, 1723 (1950).

\section{Fat Oxidation and Hæmatin Systems in Cheese}

Mock work has been done in New Zealand on a defect of Cheddar cheese characterized by a coupled oxidation of the fat and carotene. The defect, commonly known as 'tallowy discoloration' or 'white streak', is seen in the interior of the cheese as bleached areas having a tallowy flavour. These bleached areas are invariably associated with cracks in the body of the cheese. The defect usually occurs only after at least 16-20 weeks storage at low temperatures (42-44 ${ }^{\circ}$ F.). Previous attempts to find the major pre-disposing cause of this defect have not been completely successful.

As I believe the defect to be similar in several ways to oxidative changes described by Tappel $^{1}$, attempts were made to show the presence of a fatoxidizing system in cheese, using methods similar to those of Blain and Todd ${ }^{2}$. Such a system was found to be present in some cheese. As a result of these findings, cheese was made in which sterile, defibrinated cow's blood was added to the cheese milk. The experimental and control cheese were stored in a controlled curing room at about $55^{\circ} \mathrm{F}$. for 6 weeks. Sample plugs of the cheese were then stored in the presence of air, at $32-34^{\circ} \mathrm{F}$. for 4 weeks.
After this time, the cheese containing the highest amount of blood (0.02 per cent in the cheese milk) had developed typical tallowy discoloration. The control cheese containing no added blood had not devoloped the defect; it was of normal flavour and colour.

On obtaining these results, four of the original experimental and control cheeses were cut and examined. These cheeses had been at $55^{\circ} \mathrm{F}$. for 2 weeks followed by $9 \frac{1}{2}$ weeks at $42-44^{\circ} \mathrm{F}$. - a total of $11 \frac{1}{2}$ weeks. Tallowy discoloration was seen in the two experimental cheeses, being more extensive in the cheese containing the higher amount of blood. The two control cheeses showed no sign of the defect.

The possibility of abnormal amounts of blood in the milk being the prime cause of tallowy discoloration must now be considered. It has been shown that, during manufacture of experimental cheese, most of any red blood cells added to the milk become concentrated within the cheese curd. This concentration is further modified by the addition of salt to the curd, resulting in a partial hæmolysis of the red blood cells. Higher concentrations of salt would then be expected to reduce tallowy discoloration. The effect of added salt provides a possible partial explanation of the results obtained by Bishop (preceding communication).

Full details of these and related experiments will be offered for publication elsewhere.

\section{G. RammelL}

1 Tappel, A. L., Food Res., 18, 572 (1953).

${ }^{3}$ Blain, J. A, and Todd, J. P., J. Sci. Food Agric., 9, 235 (1958).

\title{
NON-INVERTED VERSUS INVERTED PLOTS IN ENZYME KINETICS
}

$I^{\mathrm{T}}$ was first shown, but not published, by Woolf (see ref. 1) that the Michaelis-Menten equation $v=V_{m} /\left(1+K_{m} / S\right)$, relating the initial reaction-rate $(v)$ of an enzymic reaction to the substrate concentration $(S)$ can be written in three linear forms. The experimental (apparent) kinetic constants $V_{m}(v$, when $S \rightarrow \infty)$ and $K_{M}\left(S\right.$, when $\left.v=V_{m} / 2\right)$ can be obtained from plots of the variables $1 / v$ versus $1 / S$, $S / v$ versus $S$ or $v$ versus $v / S$. Since its first application by Lineweaver and $B_{\text {urk }}^{2}$, the $1 / v$ versus $1 / S$ plot has been used most commonly.

However, it has been pointed out on several occasions (see ref. 3 ), that the $v$ versus $v / S$ plot, non-inverted with respect to $v$, has advantages over the two inverted plots, partly because it is less apt to obscure deviations from linearity. With respect to similar plots for determining the number of molecules of a small molecular compound bound to a protein, Scatchard" states: ". . this [inverted plot] has the disadvantage of concealing deviations from the ideal laws and of tempting straight lines where there should be curvature".

These considerations apply also to the determination of kinetic constants from enzymic reaction-rates at constant substrate concentration and varying concentrations of an inhibitor or activator (see ref. 5).

Despite the drawbacks it has remained customary, at least in enzyme kinetics, to use inverted plots in the evaluation of results. Even in a recent and authoritative text-book on enzymology that treats these graphical procedures extensively, the advan- tages of the $v$ versus $v / S$ plot have not been fully recognized. For these reasons some further comment on this matter seems to be appropriate.

In an acid-base titration, or in the demonstration of the influence of the hydrogen ion concentration on the rate of an enzymic reaction, unwieldy graphs would be obtained if instead of $p \mathrm{H}$ the hydrogen ion concentration were used as one of the co-ordinates. This applies also to the influence of the substrate concentration on the rate of an enzymic reaction, and accordingly plots of $v$ versus $p S$ have been used in enzyme kinetics. The inflexion point of the theoretical sigmoid curve corresponds to $p K_{M}$.

Like other titrations, the enzyme-substrate 'titration' should be carried out over a range that includes substrate concentrations above as well as below this inflexion ('halfway') point. An ideal range is that of about one $p S$ unit below to one $p S$ unit above this point, that is, from about $0 \cdot 1$ to $10 K_{M^{5,7}}$, representing roughly the range of 10-90 per cent 'saturation' of enzyme with substrate. However, such a (semi) logarithmic plot is still of little value for the estima. tion of the kinetic constants because it is not linear. On this basis, the advantage of the $v$ versus $v / S$ plot becomes immediately apparent. It can be seen in Fig. 1 that for almost 80 per cent of its course this plot nearly coincides with the $v$ versus $p S$ plot. It has the additional advantage over the latter of being linear, which allows $V_{m}$ to be determined by extrapolation to $S \rightarrow \infty$ (intercept with the ordinate). The (negative) slope is equal to $K_{M}$. 